\title{
Detraining Effects on Musculoskeletal Parameters in Early Postmenopausal Osteopenic Women: 3-Month Follow-Up of the Randomized Controlled ACTLIFE Study
}

\author{
Wolfgang Kemmler ${ }^{1}$ (D) Michael Hettchen ${ }^{1} \cdot$ Matthias Kohl $^{2} \cdot$ Marie Murphy $^{3} \cdot$ Laura Bragonzoni $^{4} \cdot$ Mikko Julin $^{5}$. \\ Tapani Risto $^{5} \cdot$ Simon von Stengel ${ }^{1}$
}

Received: 5 November 2020 / Accepted: 19 February 2021 / Published online: 12 March 2021

(c) The Author(s) 2021

\begin{abstract}
Periods of absence from supervised group exercise while maintaining physical activity might be a frequent pattern in adults' exercise habits. The aim of the present study was to determine detraining effects on musculoskeletal outcomes after a 3-month detraining period in early post-menopausal, osteopenic women. Due to the COVID-19 pandemic, we terminated the 18-month randomized controlled ACTLIFE exercise intervention immediately after the 13-month follow-up assessment. This put an abrupt stop to the high-intensity aerobic and resistance group exercise sessions undertaken three times per week by the exercise group (EG: $n=27$ ) and the gentle exercise program performed once per week for the attention control group (CG: $n=27$ ); but both groups were permitted to conduct individual outdoor activity for the 3-month lock-down period. Study endpoints were lean body mass (LBM), bone mineral density (BMD) at the lumbar spine (LS), maximum hip-/leg extension strength and power. Detraining-induced reductions of LBM, hip/leg strength and power (but not BMD-LS) were significantly greater $(p<0.001$ to $p=0.044)$ compared with the CG. Significant exercise effects, i.e. differences between EG and CG, present after 13 months of exercise, were lost after 3 months of detraining for LBM $(p=0.157)$ and BMD-LS $(p=0.065)$, but not for strength $(p<0.001)$ and power $(p<0.001)$. Of note, self-reported individual outdoor activities and exercise increased by about $40 \%$ in both groups during the lock-down period. Three months' absence from a supervised group exercise protocol resulted in considerable detraining effects for musculoskeletal parameters. Thus, exercise programs for adults should be continuous rather than intermittent.
\end{abstract}

Trial registration number: ClinicalTrials.gov: NCT04420806, 06.05.2020.

Keywords Detraining $\cdot$ High-intensity exercise $\cdot$ Bone mineral density $\cdot$ Lean body mass $\cdot$ Strength and power

\section{Introduction}

Wolfgang Kemmler

wolfgang.kemmler@imp.uni-erlangen.de

1 Institute of Medical Physics, Friedrich-Alexander University Erlangen-Nürnberg (FAU), Henkestrasse 91, 91052 Erlangen, Germany

2 Department of Medical and Life Sciences, University of Furtwangen, Schwenningen, Germany

3 Doctoral College, Ulster University, Newtownabbey, Co. Antrim, Northern Ireland, UK

4 University of Bologna, Campus Rimini, Rimini, Italy

5 Laurea University of Applied Sciences, Espoo, Finland
Regular exercise is thought to mitigate negative consequences of the menopausal transition which considerably affect women's lives. In the ACTLIFE study $[1,2]$ canceled prematurely due to COVID-19, we demonstrated significant positive effects of a 13-month supervised exercise program on body composition, Bone Mineral Density, menopausal symptoms and physical fitness. The three-month lock down of all training facilities in Bavaria, Germany provided an opportunity to examine the effect of a short break in a structured group exercise programs on these parameters. Due to the large number of health care providers and commercial suppliers who do not provide continuous exercise programs, this issue is highly relevant. In a recent 16-week study, on younger (20-35 years) and older adults (60-75 years), Bickel 
et al. [3], reported significant reductions of lean body mass after only 8 weeks of detraining. Several studies confirmed the deleterious effects of short-moderate training breaks/ detraining after aerobic, resistance or concurrent exercise in non-athletic cohorts. However, most of these studies (a) focus on people 60 years and older (e.g. [4-12], and/or applied (b) short training periods ${ }^{1}$ (e.g. $[6,11,13,14]$ and/ or (c) long detraining periods (i.e. 24 weeks and longer, e.g. $[10,15-18])$, the latter particularly refer to detraining studies on bone mineral density (BMD) (e.g. [19-21].

We aimed to determine the effect of moderate periods of absence from intense multimodal group exercise (3 months) on musculoskeletal parameters in a cohort of early postmenopausal women.

Our main hypotheses are 3 months of detraining after 13 months of exercise leads to significantly higher reductions of (a) lean body mass (primary hypothesis), (b) BMD at the lumbar spine (LS), (c) maximum hip/leg extension strength and (d) power compared with an attention control group (i.e. differences in detraining changes between the EG and the CG).

We further hypothesize that after 13 months of training and 3 months of detraining, the significant training effects for the parameters $(\mathrm{a}-\mathrm{d})$ are lost (i.e. difference in overall changes from baseline to 16 month follow-up between the EG vs. the CG.)

\section{Methods}

The present work is part of the ACTLIFE project, a European Project that focuses on the development and dissemination of best practice exercise protocols for therapy and prevention of osteoporosis. ACTLIFE was designed as an 18-month multimodal randomized control exercise trial that addressed the menopausal risk factor with specific regard to musculoskeletal parameters. However, due to the COVID19-induced lockdown of all training facilities as of March 17th 2020, ACTLIFE had to stop immediately after the 13-month follow-up assessment. After approval from the FAU Ethics Committee (number 118_18b), informed consent of all study participants and study registration (ClinicalTrials.gov: NCT04420806), the 3-month (detraining) follow-up assessment was conducted in mid-June 2020, one week after the reopening of assessment and training facilities (June 8th, 2020).

$1 \ldots 8-12$ weeks, i.e. a period that might be too short to determine the full amount of adaptation to exercise.

\section{Participants}

The recruitment process of the ACTLIFE-RCT has already been reported in detail. In summary, women were eligible if they met the following criteria: (a) 48-60 years old, (b) early-menopause status (1-5 years of amenorrhea), (c) osteopenia or osteoporosis at the LS, femoral neck (FN) or total hip (TH), (d) no medication, conditions and diseases known to affect bone metabolism or contraindicate group exercise or tests, (e) no high impact or resistance exercise in the past 5 years, (f) no secondary osteoporosis or osteoporotic fractures, $(\mathrm{g})$ no acute or recent history of cancer (last 5 years), (h) alcohol consumption $<60 \mathrm{~g} / \mathrm{d}$ on 5 days/ week) (Fig. 1). Applying these criteria 54 women eligible and willing to participate were randomly assigned to the two study groups (Fig. 1).

\section{Randomization Procedures}

Briefly, participants stratified for LS-BMD were randomly allocated to exercise $(n=27)$ and control group $(n=27)$ by drawing lots. Neither researchers nor participants knew the allocation beforehand ("allocation concealment").

\section{Blinding}

The blinding strategy of ACTLIFE included outcome assessors and test assistants who were unaware of and not allowed to ask the participants' group assignment (EG or CG).

\section{Study Procedure}

Apart from the high-intensity multimodal exercise protocol for the EG and the low intensity/low volume program for the $\mathrm{CG}$, all participants were provided with cholecalciferol (VitD) and calcium (Ca) supplements according to recent recommendations (i.e. 800 IU/day Vit-D; $1000 \mathrm{mg}$ /day Ca [22], details see below). In contrast to the exercise protocol that had to be stopped in mid-March 2020, calcium and Vit-D was provided up to study end in mid-June 2020. Apart from the interventions, participants were requested to maintain their usual dietary intake and lifestyle including habitual physical activity and exercise habits.

\section{Intervention}

\section{Exercise Intervention}

All EG and CG participants started the intervention in MidFebruary 2019 and had to stop exercising in Mid-March 2020 due to the corona-induced lockdown of all training facilities in Bavaria, Germany. 
Fig. 1 Participant flow through the ACTLIFE study

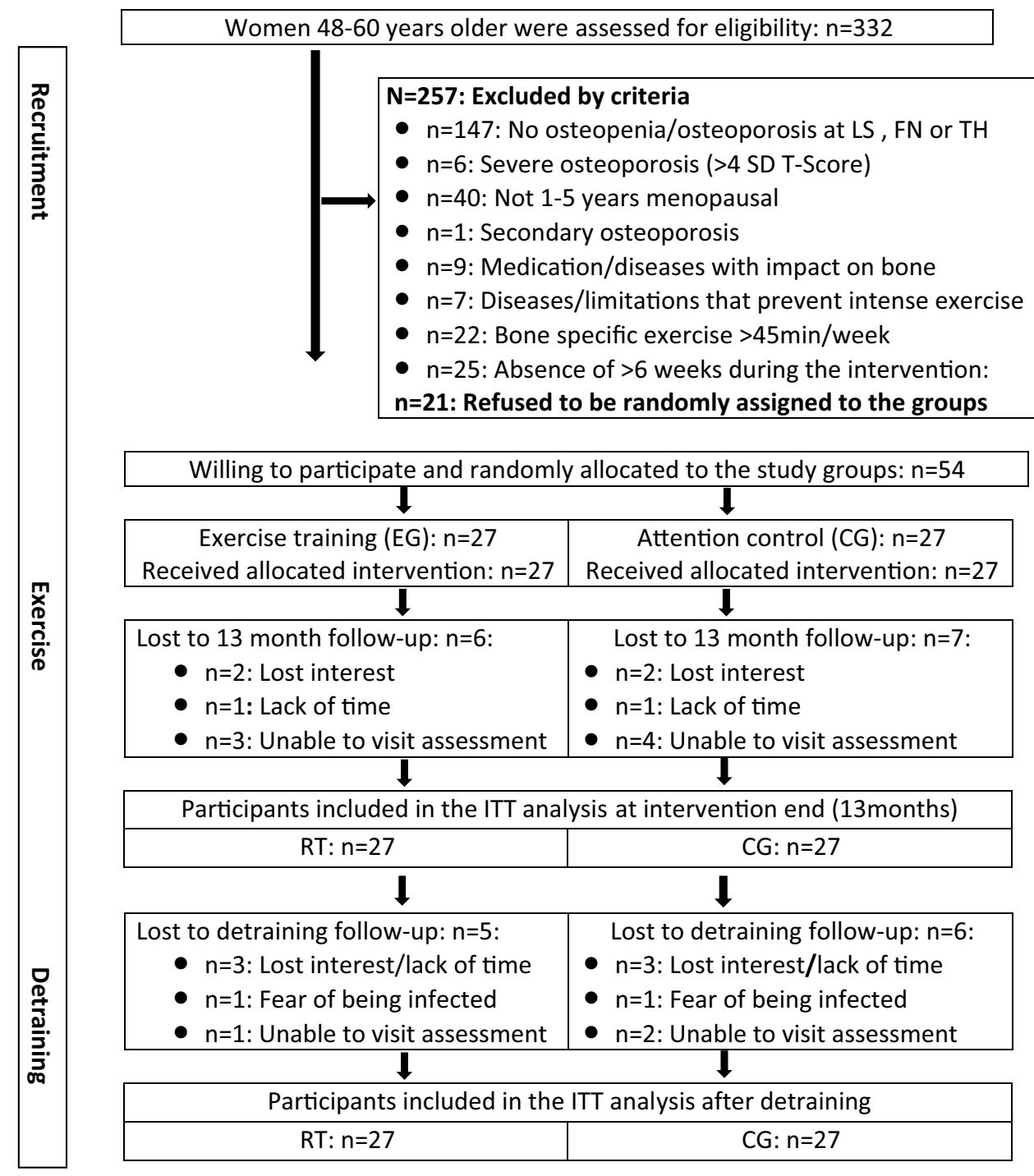

\section{Exercise Group}

The EG exercise protocol has been described in detail previously $[1,2]$; thus we only outline it here. In summary, our exercise protocol can be considered as a periodized high-intensity approach that focused on musculoskeletal parameters. In general, we structured our exercise protocol into 8-12-week blocks of high-intensity/high-effort exercise, interspersed by $4-5$ weeks of lower exercise intensity/ volume. Applying consistently 3 sessions/week we realized 15-20 min of aerobic dance in a high-intensity interval training (HIIT) mode $^{2}$ with moderate to high ground reaction

\footnotetext{
$220 \mathrm{~min}$ of aerobic dance; after $5 \mathrm{~min}$ of warm up either $10 \times 60 \mathrm{~s}$ at $85 \%$ HFmax interspersed by $60 \mathrm{~s}$ of lower exercise intensity or a corresponding $30 \mathrm{~s} / 30 \mathrm{~s}$ protocol was applied.
}

forces (GRF), ${ }^{3}$ jumping $^{4}$ and periodized high intensity (60-85\% $1 \mathrm{RM}$ )/ high effort $^{5}$ resistance training (HIT-RT) [24]. The two sessions conducted in our lab focused on HIIT and HIT-RT, the latter in a single set circuit mode using barbells/body weight. The third session was conducted in a multiple set mode on resistance exercise machines in a dedicated gym. Independently of the setting, RT-exercises (10-14 exercises) addressed all the main muscle groups. Apart from high intensity/effort, we manipulated movement velocity during RT by applying periods of explosive movements

\footnotetext{
${ }^{3}$ Up to 120 movements/session with GRF: $2.0-3.0 \times$ body mass.

${ }^{4}$ Up to $50-60$ bilateral jumps/session with GRF up to $4-4.5 \times$ body weight.

5 Applying "work to repetition maximum" i.e. set endpoint when trainees complete the final repetition possible whereby if the next repetition were attempted they would definitely achieve muscular failure. [23] and intensifying strategies (i.e. supersets, drop-sets) [24]
} 
Table 1 Baseline and 13-month follow-up characteristics of the ACTLIFE-RCT study

\begin{tabular}{|c|c|c|c|c|}
\hline \multirow[t]{2}{*}{ Variable } & \multicolumn{2}{|l|}{$\begin{array}{l}\mathrm{CG}(n=27) \\
\mathrm{MV} \pm \mathrm{SD}\end{array}$} & \multicolumn{2}{|l|}{$\begin{array}{l}E G(n=27) \\
M V \pm S D\end{array}$} \\
\hline & Baseline & 13 months & Baseline & 13 months \\
\hline Age [years] & $54.5 \pm 1.6$ & $55.6 \pm 1.6$ & $53.6 \pm 2.0$ & $54.6 \pm 2.0$ \\
\hline Body height $[\mathrm{cm}]$ & $164.5 \pm 8.2$ & $164.5 \pm 8.2$ & $164.2 \pm 6.0$ & $164.2 \pm 6$ \\
\hline Body mass $[\mathrm{kg}]$ & $67.4 \pm 14.6$ & $68.4 \pm 14.1$ & $64.0 \pm 9.6$ & $63.7 \pm 10.2$ \\
\hline Calcium intake [mg/day] & $642 \pm 265$ & $671 \pm 301$ & $645 \pm 252$ & $666 \pm 274$ \\
\hline Vit-D level (25-OHD) [ng/ml] & $21.6 \pm 10.8$ & $30.7 \pm 11.6$ & $27.8 \pm 11.7$ & $37.1 \pm 12.8$ \\
\hline Years after menopause [year] & $3.5 \pm 1.1$ & $4.5 \pm 1.1$ & $3.7 \pm 1.0$ & $4.8 \pm 1.0$ \\
\hline Exercise volume [min/week] & $46 \pm 38$ & $51 \pm 45$ & $64 \pm 48$ & $57 \pm 42$ \\
\hline Individual outdoor activity [min/week] & $110 \pm 74$ & $119 \pm 90$ & $131 \pm 94$ & $126 \pm 88$ \\
\hline Waist circumference $[\mathrm{cm}]$ & $91.1 \pm 9.9$ & $90.8 \pm 9.7$ & $87.8 \pm 8.6$ & $86.0 \pm 8.7$ \\
\hline Energy intake ${ }^{\mathrm{a}}[\mathrm{kcal} / \mathrm{day}]$ & $2067 \pm 355$ & $2088 \pm 387$ & $2009 \pm 444$ & $2051 \pm 403$ \\
\hline Protein intake $[\mathrm{g} / \mathrm{kg} /$ body mass/day] & $1.20 \pm 0.21$ & $1.16 \pm 0.19$ & $1.18 \pm 0.27$ & $1.21 \pm 0.23$ \\
\hline Ovariectomy $<50$ years $[n]$ & 0 & - & $1^{\mathrm{b}}$ & - \\
\hline Family disposition $^{\mathrm{c}}[n]$ & 9 & - & 7 & - \\
\hline
\end{tabular}

${ }^{\mathrm{a}}$ As determined by a 4-day dietary protocol, see methods

${ }^{\mathrm{b}}$ At age 47 years

${ }^{\mathrm{c}}$ Fragility fractures or verified osteoporosis in close relatives (parents, aunts, uncles, grandparents) in the concentric phase. The consistently supervised 8-12week phases were structured into 2-3 linearly periodized 4-week periods with each 4th week as a regeneration week with lower intensity (60-65\% 1 RM) and lower effort (nonrepetition maximum). During the 4-5-week intermitted periods, one circuit session (60-75\% $1 \mathrm{RM}$, however not to RM [23]), one 45 min session of stretching and easy floor exercises (see control group) and one 15 min video-guided home training session (see control group) were scheduled.

\section{Control Group}

Exercise in the attention control group focused on stability, flexibility and well-being, albeit with strong emphasis on applying an exercise protocol unlikely to affect "bone", "body composition" or "maximum strength/power". During the 13-month intervention period, we completed two cycles of 12 weeks with one session of $45 \mathrm{~min} /$ week of consistently supervised group exercises intermitted by 12 and 14 weeks of non-supervised, video-guided home exercise (15 min).

\section{Vitamin-D and Calcium Supplementation}

All participants were provided with 5000 IE/week of cholecalciferol (MYPROTEIN, Cheshire, UK) [22], independently of their baseline 25OH D levels (Table 1). Based on dietary calcium intake ("calcium questionnaire", Rheumaliga, Switzerland), we supplemented calcium carbonate capsules (Sankt Bernhard, Bad Dietzenbach, Germany) to realize a calcium intake of $1000 \mathrm{mg} /$ day [22].

\section{Compliance with the Exercise Intervention}

Instructors and chip card systems were used to monitor participants' exercise attendance. Adherence to the exercise protocol, particularly for exercise intensity, was checked (1) by the instructors monitoring the load/repetition proportion during the sessions and (when necessary) asking participants to work with more effort and (2) by reviewing the participants' training logs after the 8-12week meso-cycles.

\section{Study Outcomes}

\section{Primary Study Outcome(s)}

- Changes of (soft) lean body mass determined by dualenergy X-ray absorptiometry (DXA) from intervention end (13 months) to 3-month detraining follow-up.

\section{Secondary Study Outcomes}

- Changes of BMD at the lumbar spine as determined by DXA from intervention end (13 months) to 3-month detraining follow-up.

- Maximum dynamic hip-/leg-extension strength changes as determined by an isokinetic leg press from intervention end (13 months) to 3-month detraining follow-up. 
- Maximum dynamic hip-/leg-extension power (“jumping height") as determined by a force plate from intervention end (13 months) to 3-month detraining followup.

\section{Changes of Trial Outcomes After Trial Commencement}

Due to the rapid and strict COVID-19 lockdown, we were unable to repeat the MRI assessment at intervention end (13 months) and correspondingly did not apply MRI after the detraining period (i.e. 3-month follow-up).

\section{Assessments}

Standardized testing and assessments were used at each timepoint. Participants were briefed and asked to avoid changes in dietary intake or high physical activity $48 \mathrm{~h}$ prior to the tests. All the tests were conducted at about the same time of day ( $\pm 90 \mathrm{~min})$, using the same calibrated devices and/or specifications and the same protocols, but not always (i.e. strength/power assessments) by the same test assistant.

Body height was determined using a Holtain stadiometer (Crymych Dyfed., Great Britain). Body mass was assessed using direct-segmental, multi-frequency Bio-ImpedanceAnalysis (DSM-BIA; InBody 770, Seoul, Korea), which was also used as a backup assessment for body composition (results not reported here). Areal BMD and body composition were assessed by DXA (QDR 4500a, Discoveryupgrade, Hologic Inc., Bedford, USA). Regions of interest on BMD and regional body composition was segmented using the "compare mode", so that area and placement of the baseline assessment were reproduced exactly during all the FU assessments.

Maximum isokinetic leg-/hip-extensor strength was assessed with an isokinetic leg press (CON-TREX LP, Physiomed, Laipersdorf, Germany). The range of motion was $30^{\circ}-90^{\circ}$ within the knee angle; velocity of the movement was $0.2 \mathrm{~m} / \mathrm{s}$. After familiarization with the testing procedure five reps with maximum effort ("push as strongly as possible") were conducted.

Lower extremity power was determined by a countermovement jump (CMJ) with hands on hips (i.e. no arm swing). Participants were asked to "jump as high as possible" starting from an upright position. Participants were requested to maintain extension in the hip, knee, and ankle joints after take-off to prevent any additional flight time by flexing their legs during landing. Tests were conducted on a force platform (KMP Newton GmbH, Stein, Germany). The jumping height was calculated automatically by the software provided by the manufacturer based on ground reaction forces.
A standardized baseline questionnaire [25], a 4-day dietary protocol (Freiburger Ernährungsprotokoll, see below) and specific physical activity and exercise questionnaire [26-28] asked for (a) demographic parameters; (b) diseases, physical limitations and pharmacologic therapy under special consideration of osteoporosis risk and ability to frequently conduct intensive exercise; (c) dietary supplements; (d) pain frequency and severity at the lumbar spine region [29] and (e) lifestyle, including physical activity and exercise [26-28].

During follow-up (FU) the same questionnaires aimed to evaluate changes from baseline, additionally we also asked for changes of lifestyle, diet, exercise, and pain levels in the FU questionnaire. This questionnaire focused mainly on changes in pharmacologic therapy, diseases or operations i.e. also parameters that might have affected the present study outcomes. Of key importance for reliable results, we placed strong emphasis on consistency, completeness and accuracy by checking the completed questionnaires together with the participants.

Dietary intake was recorded on 3 weekdays and one weekend day characteristic for dietary habits at baseline and after 7 months, 13 months and 16 months. Participants were provided with simple diet records (Freiburger Nutrition Record, nutri-science, Hausach, Germany) which were analyzed consistently by the same research assistant. In cases of unlikely results, (e.g. energy intake $<1000 \mathrm{kcal} /$ day or $>3500 \mathrm{kcal} / \mathrm{day}$ ), the women were requested to provide another dietary record based on more representative days.

\section{Sample Size Calculation}

The initial sample size calculation was based on "BMD changes at the LS" after 18 months. In order to generate an estimated effect ( $\Delta$-EG vs $\Delta$-CG) on BMD-LS of $2.0 \pm 2.5 \%$ $[30,31]$, the sample size required to generate $80 \%$ power $(1-\beta)$ and alpha $=0.05$ was 25 participants per group. We included 27 participants to allow for drop-outs within an additional per protocol analysis for the primary study outcome.

\section{Statistical Analysis}

We conducted an intention to treat (ITT) analysis that included all participants initially assigned to the EG and CG. ITT was performed using R statistics software [32], in combination with Amelia II [33]. The full data set was used for multiple imputations. Imputation was repeated 100 times. According to imputation diagnostic plots, imputation worked well. In addition to the ITT analysis, we conducted a per protocol analysis for the primary hypothesis, that included all participants with 13- and 16-month data, independently of their compliance. Normal 


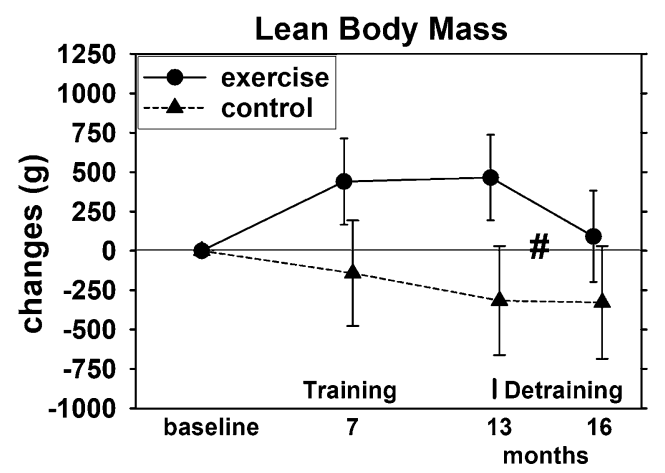

Fig. 2 Mean values and 95\% CI for changes of Lean Body Mass (LBM) after training and detraining based on the intention to treat analysis $(n=27$ in the EG and CG). \# significant difference in changes from 13-month (end of intervention) to 3-month $\mathrm{FU}$ (16 month) in the EG vs. CG

distribution of the study endpoints was checked by statistical (Shapiro-Wilks) and graphical (qq-plots) procedures. In order to compare changes for the detraining period between the EG and the CG (primary hypothesis) we applied an ANCOVA adjusted for 13-month data. In parallel, to determine overall effects after training and detraining (secondary hypothesis), we also applied an ANCOVA that adjusted on baseline data. Finally the changes over time inside the groups i.e. training (baseline to 3 months) and detraining effects (13-16 months) were investigated by paired $t$ tests applying the approach of Barnard and Rubin [34]. Two-tailed tests were applied and significance was accepted at $p<0.05$.

\section{Results}

Participants' baseline and 13-month characteristics are displayed in Table 1. Albeit non-significant, 25-OHD levels vary considerably, while other participant characteristics at baseline and intervention end (13 months) were similarly distributed.

In summary, five women in the EG and six women of the CG were lost to 16-month follow-up (Fig. 1). Altogether six participants quit during the intervention period. With respect to the 3-month detraining follow-up, two participants refused to be assessed due to fear of being infected, two other women were unable to visit the assessments (Fig. 1).

Attendance rate was $79 \pm 12 \%$ in the $E G$ and $78 \pm 14 \%$ in the CG. The monitoring of "effort" by checking the relationship of reps and load selected to realize RM specification indicates that in one fourth to one third of the cases women did not follow our RM prescription. This refers particularly to the first 12-week mesocycle.

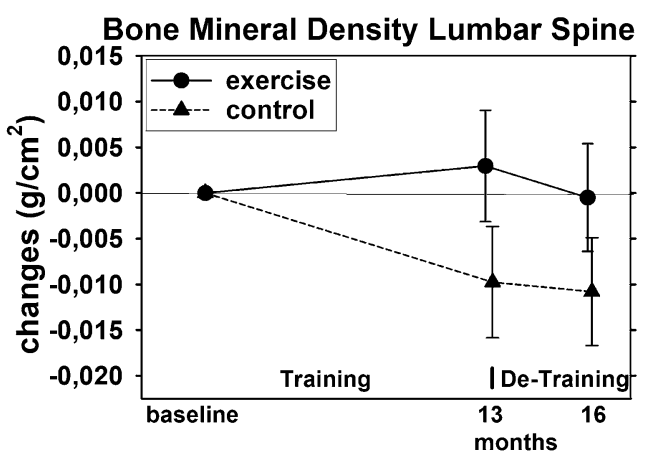

Fig. 3 Mean values and 95\% CI for changes of BMD at the lumbar spine after training and detraining based on the intention to treat analysis ( $n=27$ in the EG and CG)

\section{Primary and Secondary Study Outcomes}

During the intervention period, LBM increased significantly in the EG $(p<0.001)$ and decreased non-significantly $(p=0.106)$ in the CG (Fig. 2), resulting in significant $(p<0.001)$ exercise effects after 13 months of exercise. However, with respect to the primary hypothesis, LBM reduction during the detraining phase was significant for the EG $(p=0.019)$, while no relevant changes were observed for the CG. Most importantly, however, we found significant differences $(p=0.044)$ in LBM changes (study end -3 month FU) between the EG and the CG (primary hypothesis). Results of the ITT-analysis with respect to the primary hypothesis were supported by the per protocol analysis. Moreover, after 16 months, no more significant exercise effects (baseline to 16 months) on LBM were observed $(p=0.157)$ (secondary hypothesis).

Accordingly, we accepted the main hypothesis (a) that 3 months of detraining after 13 months of exercise lead to significant higher reductions of lean body mass compared to an attention control group and (subordinate hypothesis) that exercise effects on LBM observed at intervention end (13 months) were lost after 3 months of detraining (16 month).

After 13 months of intervention, significant BMD reductions were determined for the CG $(p=0.004)$ and slight increases were observed in the EG $(p=0.337)$. In summary, exercise effects (EG vs. CG) after 13 months were significant $(p=0.027)$. However, more importantly three months of detraining reduced LS-BMD in the EG slightly below baseline values although the reduction was not significant $(p=0.115)$, while no relevant negative BMD change during the detraining phase was observed for the CG. Nevertheless, changes during the detraining phase did not differ significantly between the two groups $(p=0.523)$. This result is confirmed by the per protocol analysis $(p=0.477)$. Moreover, the overall effect after 


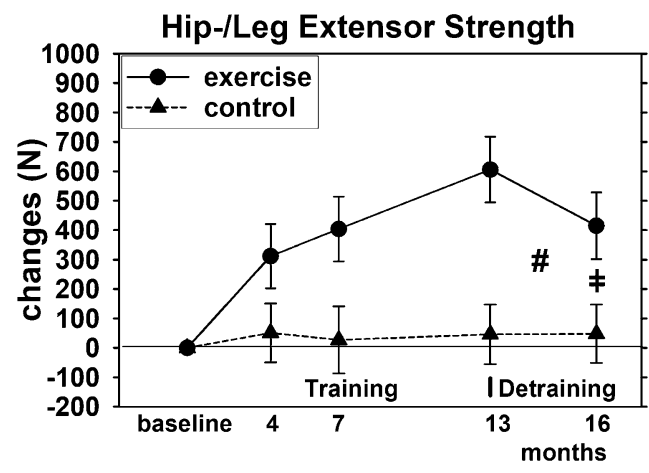

Fig. 4 Mean values and 95\% CI for changes of hip-leg extensor strength after training and detraining based on the intention to treat analysis $(n=27$ in the EG and CG). \# significant difference in changes from 13-month (end of intervention) to 3-month FU (16 month) in the EG vs. CG. ₹ significant different changes from baseline to 16 month (i.e. "overall effects")

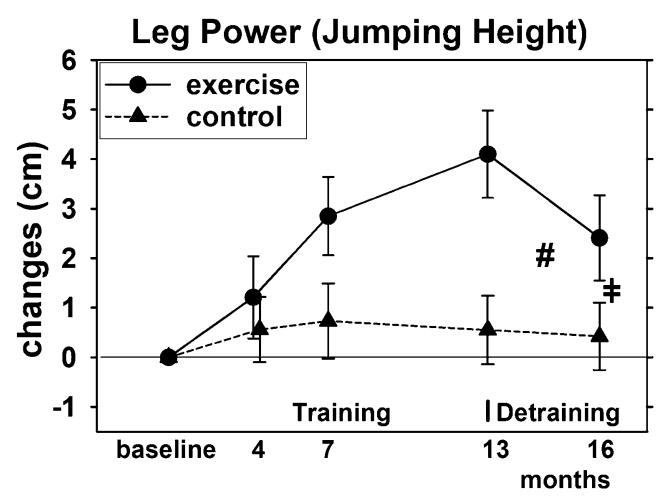

Fig. 5 Mean values and 95\% CI for changes of leg power after training and detraining based on the intention to treat analysis $(n=27$ in the EG and CG). \# significant difference in changes from 13-month (end of intervention) to 3-month FU (16 month) in the EG vs. CG. ₹ significant different changes from baseline to 16 month (i.e. "overall effects")

16 months of training and detraining was non-significant $(p=0.065)$ (Fig. 3).

With respect to BMD at the LS, we revised our hypothesis (b) that 3 months of detraining after 13 months of exercise leads to significant higher reductions of BMD-LS compared to an attention control group; however, we confirmed that exercise effects for BMD observed at intervention end (13 months) were lost after 3 months of detraining.

Maximum hip-/leg-extension strength and power of the EG increased significantly during the intervention phase (both $p=0.001$ ) and increased slightly (significant for 7-month FU) in the CG (Figs. 4 and 5) resulting in significant $(p<0.001)$ exercise effects after 13 months of exercise.

Strength and power reductions during the detraining phase were significant for the EG (both $p<0.0 .001$ ), and differ significantly (both $p<0.001$ ) from changes in the CG. The latter result was confirmed by the additional per protocol analysis. After 16 months, i.e. 13 months of training and 3 months of detraining, the exercise effect (between group difference) was still significant (both $p<0.001$ ) (Figs. 4 and 5).

Thus, we confirmed hypotheses (c) and (d) that 3 months of detraining lead to significant higher reductions of maximum hip-/leg-extension strength and power compared to an attention control group. However, we revised our hypotheses that significant exercise effects were lost during the detraining period.

\section{Confounding Parameters}

During the detraining period no relevant changes or between group differences for dietary intake parameters (i.e. energy, carbohydrate, fat, protein, alcohol intake), pharmacologic therapy or diseases were observed. Habitual physical activity was maintained in both groups, however, as determined by a dedicated questionnaire, the volume of individual aerobic outdoor activities (i.e. brisk walking, cycling, jogging) increased significantly ( $p<0.001)$ in both groups by $41 \%$ (EG) and 37\% (CG). In contrast, although most women worked from home ("home office") during the lockdown, relevant changes of occupational physical activity were not reported. Of further interest, all but four participants of the EG and CG each conducted the $15 \mathrm{~min}$ exercise video at least once per week (EG: $1.4 \pm 0.7$ vs. CG: $1.7 \pm 1.0$ sessions/week).

\section{Discussion}

In this study, we clearly confirmed the deleterious effect of short-moderate term detraining periods on musculoskeletal parameters and menopausal complaints in early postmenopausal women. Of note, participants were not physically inactive during the ACTLIFE detraining period, but significantly increased individual aerobic outdoor activities (e.g. walking) during the three months. Although we cannot determine whether this affected our results, we speculate that together with the continued application of the 15 min video-guided home training session, it might have attenuated the negative effect of detraining on our outcomes.

Reviewing our results in detail, the significant reduction of LBM to baseline values in only 3 months of absence of high-intensity aerobic and resistance exercise is particularly remarkable (Fig. 2). In one of the few comparable studies on combined aerobic and RT exercise (AS), Douda 
et al. [7] applied four 9-month exercise blocks followed by 3 months of detraining each. The authors reported regular (non-significant) decreases of LBM after the training phase that consistently reach pre-training values in women 60 years + . However, apart from one 9-month block, the AS-protocol did not significantly increase LBM during the training phases. In another, albeit much shorter, trial (16 weeks), Bickel et al. [3] applied an intense multiple set RT for the lower limbs in older adults (60-75 years) ${ }^{6}$ and reported significant reductions in thigh lean mass that dropped below baseline values after only 8 weeks of detraining. Of note, one RM knee extension (KE) strength did not decrease significantly with 6 months detraining (55.6 to $50.0 \mathrm{~kg}$ ), and still significantly exceeded baseline values $(40.8 \mathrm{~kg})$. Although in parallel to LBM, maximum hip-leg strength decreased significantly in the EG of the present study and detraining changes differ significantly from the CG $(p<0.001)$, the overall effect (EG vs. CG) is still significant after 16 months of observation and maximum strength in the EG still significantly exceeded $(p<0.001)$ baseline. This might be attributed to the aspect that neuromuscular effects which contribute to strength and power are more resistant to detraining compared with hypertrophic effects [35, 36]. Consequently, this will result in higher preservation of strength/power compared to muscle mass gains during detraining (e.g. [3, 5, 12, 37, 38]). However, these data are not undisputed. In their 8-week and 16-week RT-studies on older people (70-80 years and $90 \pm 1$ years, respectively), Lovell et al. [39] and Fiatarone et al. [40] observed significant reductions in maximum leg strength (1 RM squat) after only 4 weeks of detraining-nevertheless, detraining $1 \mathrm{RM}$ still differs significantly from baseline. On the other hand, a 24-week RTprotocol with middle aged (37-44 years.) and older adults (63-78 years), [35], did not show reductions of $1 \mathrm{RM}$ and other neuromuscular parameters of leg extension strength and power ${ }^{7}$ after a 3 -week detraining phase. Therefore, apart from the length of the detraining phase and of course from training effects, ${ }^{8}$ there is some evidence that stability of adaption increases with the duration over which training is performed [41]. Reviewing studies with longer intervention periods, after 9 months of multicomponent exercise and 3 months of detraining, Esain et al. [8] reported maintained upper and lower limb strength and endurance in his cohort of adults 65 years and older. This result was

\footnotetext{
6 The authors [3] observed significant increases of TLM (4.4\%) and KE strength (36\%) after 16 weeks of training.

7 apart from a significant decrease of maximum isometric force (6\%) during unilateral leg extension in the younger subgroup.

${ }^{8}$ Detraining effects can only occur after prior training effects; correspondingly higher training effects at least offer a higher potential for detraining effects $[39,40]$.

${ }^{9}$ Chair stand, arm curl [4].
}

confirmed by Vuori et al. [42], who did not report relevant changes of maximum leg extensor strength after 3 months of detraining following a 12-month RT with young women (19-27 years). In contrast, Carvalo et al. [4], who applied a 8-month multicomponent exercise program, listed significant reductions of functional strength parameters ${ }^{9}$ after 3 months of detraining in older women (64-85 years.), although leg extension but not arm flexor strength still significantly exceeded baseline values.

Due to the need for longer training (...and detraining?) periods due to slower bone metabolism [43] and the more discreet training effects of training on bone (compared to muscle mass or strength) [44], detraining studies on BMD are rare [19-21, 42, 45, 46]. Two studies that applied similar long training (8 months and 12 months) and detraining periods ( 3 months and 4 months) $[42,46]$, albeit in young women (18-27 years.), confirmed our results. Both authors reported moderate, non-significant decreases $(\approx 1.5 \%)$ of BMD at the LS after an exercise-induced gain of 2.0\% [42] to $3-3.5 \%$ [46]. Another study with older osteosarcopenic men who also underwent a corona-induced training break after 18 months of high-intensity dynamic resistance exercise [45], reported a significant reduction of BMD-LS, muscle mass and function after 6 months of detraining. As with the present study, training-induced effects after the detraining period remained significant for muscle mass and function, but not for BMD of the LS.

In summary, it is difficult to estimate the optimum duration of a training break that might allow full regeneration/ resensitization of the given musculoskeletal parameter (i.e. strength, muscle, bone), without resulting in unintended detraining effects. With respect to bone, Saxon et al. [47] reported the most favorable effect on bone strength for intermitted rest protocols with 5 week of "time off". Conversely, regular bone resensitization cycles of 5-6 weeks after 10-12 week phases of high intensity/effort/velocity as applied in this and other studies [48-50] of ours, might prevent optimum development of muscle mass [3] and/or strength $[39,51] .{ }^{10}$ However, one has to bear in mind that in contrast to the detraining approaches cited above, we did not stop the exercise protocol abruptly but only reduced exercise intensity, type and volume of high impact and RT-exercise to a lower level during our "regeneration phases". In this context, data provided by Bickel et al. [3] indicate that reductions to one third of the initial RT exercise dose ${ }^{11}$ maintain

\footnotetext{
10 The latter review focuses on younger cohorts, however.

11 The authors [3] simply reduced the weekly exercise frequency from three to one session per week. Of note, an exercise volume reduction to one ninth of the initial dose preserved muscle hypertrophy in the young but not old, while strength gains were maintained in both groups.
} 
exercise gains in (thigh) muscle-mass and particular strength for 8 weeks and longer.

Our study has several limitations. (1) First of all, this was not a preplanned detraining study. After 4 weeks of detraining we decided to discontinue the study and focus on the present detraining issue. However, the COVID-19 lockdown of all training facilities ensured that none of the women continued the relevant components of the exercise protocol of the present study. (2) On the other hand, since the Bavarian COVID-19 regulation allowed individual outdoor activities, the (self-reported) volume of aerobic exercise as determined by questionnaire increased significantly in both groups. Further, most participants of the EG and CG reported that they conducted the 15 min exercise video at least once per week. (3) All the women were supplemented with recommended doses of cholecalciferol and calcium [22] throughout the detraining period. This increases the evidence $^{12}$ and our confidence that any reductions in musculoskeletal parameters were predominately related to detraining effects. (4) Also due to the unplanned implementation of detraining, we did not conduct a sample size analysis for detraining and so it might be underpowered for adequately addressing some of the endpoints. (5) We focused on study endpoints that demonstrated significant effects during the intervention period. Consequently, we did not include BMD at the total hip, for example, which failed to demonstrate significant positive effects $(p=0.129)$ after 13 months of exercise. (6) Due to the rapid and consequent lock-down and the still prevalent pandemic, we lost a considerable number of participants to 13-month and 16-month follow-up (Fig. 1). We applied ITT with multiple imputation; however, even the most sophisticated imputation approach does not completely reflect reality. This situation led us to conduct an additional per protocol analysis that included only participants with follow-up data.

In conclusion, despite increases in aerobic outdoor activities and home exercise, 3 months of absence from a supervised high-intensity group exercise protocol resulted in detraining effects that were significant for lean body mass, muscle strength and power. Thus, although it might be not completely legitimate to generalize our results to other age groups [3, 52], roughly in line with the present detraining literature, we confirmed the general need for continuous rather than intermitted exercise programs [7]. This however, does not contradict the application of short regeneration/resensitization phases during periodized exercise protocols.

\footnotetext{
12 Apart from the observation that no relevant changes of diseases or medication with impact on muscle or bone metabolism were determined during the detraining period.
}

Author Contributions Conceptualization: all authors significantly contribute to the conceptualization of the study. Data curation: $\mathrm{MH}$, WK. Formal analysis: MK, WK. Investigation: MH, SvS, MM, LB, MJ, TP, WK. Methodology: MH, SvS, MM, LB, MJ, TP, WK. Project administration: MH, WK. Resources: LB, WK, SvS. Supervision: MH, SvS, WK. Validation: MH, MK, WK. Writing-original draft: WK. Writing-review and editing: all authors significantly contribute to writing, review and editing.

Funding Open Access funding enabled and organized by Projekt DEAL. This study is one of the intellectual outputs of the project "ACTLIFE-Physical activity the tool to improve the quality of life in osteoporosis people" and had grant support from the European Union's Erasmus Plus Sport program under grant agreement No. 2017-2128/001-001.

Data Availability The datasets generated and/or analyzed during the current study are available from the corresponding author on reasonable request.

\section{Compliance with Ethical Standards}

Conflict of interest Wolfgang Kemmler, Michael Hettchen, Matthias Kohl, Marie Murphy, Laura Bragonzoni, Mikko Julin, Tapani Risto and Simon von Stengel declare that they have no conflict of interest.

Ethical Approval Ethics committee FAU Erlangen-Nürnberg number 118_18b.

Informed Consent Written informed consent was provided by all participants.

Open Access This article is licensed under a Creative Commons Attribution 4.0 International License, which permits use, sharing, adaptation, distribution and reproduction in any medium or format, as long as you give appropriate credit to the original author(s) and the source, provide a link to the Creative Commons licence, and indicate if changes were made. The images or other third party material in this article are included in the article's Creative Commons licence, unless indicated otherwise in a credit line to the material. If material is not included in the article's Creative Commons licence and your intended use is not permitted by statutory regulation or exceeds the permitted use, you will need to obtain permission directly from the copyright holder. To view a copy of this licence, visit http://creativecommons.org/licenses/by/4.0/.

\section{References}

1. Hettchen M, Kohl M, von Stengel S et al (2021) Changes of menopausal risk factors under specific regard of bone mineral density in early postmenopausal osteopenic women after 13 months of high-intensity exercise. The randomized controlled ACTLIFERCT. Clin Interv Aging 16:83-96

2. Kemmler W, Hettchen M, Kohl M et al (2020) Effects of high intensity exercise during early postmenopause. The randomized controlled ACTLIFE-study. J Osteopor Phys Act 8:228

3. Bickel CS, Cross JM, Bamman MM (2011) Exercise dosing to retain resistance training adaptations in young and older adults. Med Sci Sports Exerc 43:1177-1187

4. Carvalho MJ, Marques E, Mota J (2009) Training and detraining effects on functional fitness after a multicomponent training in older women. Gerontology 55:41-48 
5. Correa CS, Baroni BM, Radaelli R et al (2013) Effects of strength training and detraining on knee extensor strength, muscle volume and muscle quality in elderly women. Age (Dordr) 35:1899-1904

6. Dos Santos L, Cyrino ES, Antunes M et al (2016) Changes in phase angle and body composition induced by resistance training in older women. Eur J Clin Nutr 70:1408-1413

7. Douda HT, Kosmidou KV, Smilios I et al (2015) Communitybased training-detraining intervention in older women: a five-year follow-up study. J Aging Phys Act 23:496-512

8. Esain I, Gil SM, Bidaurrazaga-Letona I, Rodriguez-Larrad A (2019) Effects of 3 months of detraining on functional fitness and quality of life in older adults who regularly exercise. Aging Clin Exp Res 31:503-510

9. Nascimento MAD, Gerage AM, Silva D et al (2019) Effect of resistance training with different frequencies and subsequent detraining on muscle mass and appendicular lean soft tissue, IGF1, and testosterone in older women. Eur J Sport Sci 19:1-9

10. Taaffe DR, Henwood TR, Nalls MA et al (2009) Alterations in muscle attenuation following detraining and retraining in resistance-trained older adults. Gerontology 55:217-223

11. Tokmakidis SP, Kalapotharakos VI, Smilios I, Parlavantzas A (2009) Effects of detraining on muscle strength and mass after high or moderate intensity of resistance training in older adults. Clin Physiol Funct Imaging 29:316-319

12. Van Roie E, Walker S, Van Driessche $S$ et al (2017) Training load does not affect detraining's effect on muscle volume, muscle strength and functional capacity among older adults. Exp Gerontol 98:30-37

13. de Souza BE, Diefenthaeler F, Sakugawa RL et al (2019) Effects of different strength training volumes and subsequent detraining on strength performance in aging adults. J Bodyw Mov Ther 23:466-472

14. Elliott KJ, Sale C, Cable NT (2002) Effects of resistance training and detraining on muscle strength and blood lipid profiles in postmenopausal women. Br J Sports Med 36:340-344

15. Henwood TR, Taaffe DR (2008) Detraining and retraining in older adults following long-term muscle power or muscle strength specific training. J Gerontol A 63:751-758

16. Rossi FE, Diniz TA, Neves LM et al (2017) The beneficial effects of aerobic and concurrent training on metabolic profile and body composition after detraining: a 1-year follow-up in postmenopausal women. Eur J Clin Nutr 71:638-645

17. Porter MM, Nelson ME, Singh MAF et al (2002) Effects of longterm resistance training and detraining on strength and physical activity in older women. JAPA 10:260-270

18. Snijders T, Leenders M, de Groot L et al (2019) Muscle mass and strength gains following 6 months of resistance type exercise training are only partly preserved within one year with autonomous exercise continuation in older adults. Exp Gerontol 121:71-78

19. Dalsky GP, Stocke KS, Ehsani AA et al (1988) Weight-bearing exercise training and lumbar bone mineral content in postmenopausal women. Ann Intern Med 108:824-828

20. Iwamoto J, Takeda T, Ichimura S (2001) Effects of exercise training and detraining on bone mineral density in postmenopausal women with osteoporosis. J Orthop Sci 6:128-132

21. Winters KM, Snow CM (2000) Detraining reverses positive effects of exercise on the muscoskeletal system in premenopausal women. J Bone Miner Res 15:2495-2503

22. DVO (2017) Leitlinie prophylaxe, diagnostik und therapie der osteoporose bei postmenopausalen Frauen und bei Männern. Schattauer-Verlag, Stuttgart

23. Steele J, Fisher J, Giessing J, Gentil P (2017) Clarity in reporting terminology and definitions of set end points in resistance training. Muscle Nerve 10:368-374
24. Gießing J (2008) HIT-Hochintensitätstraining. Novagenics-Verlag, Arnsberg

25. Kemmler W, Lauber D, Weineck J et al (2004) Benefits of 2 years of intense exercise on bone density, physical fitness, and blood lipids in early postmenopausal osteopenic women: results of the Erlangen Fitness Osteoporosis Prevention Study (EFOPS). Arch Intern Med 164:1084-1091

26. Kemmler W, Bebenek M, von Stengel S, Bauer J (2014) Peakbone mass development in young adults: effects of study program related levels of occupational and leisure time physical activity and exercise. A prospective 5-year study. Osteoporos Int 26:653-662

27. Kemmler W, Weineck J, Kalender WA, Engelke K (2004) The effect of habitual physical activity, non-athletic exercise, muscle strength, and VO2max on bone mineral density is rather low in early postmenopausal osteopenic women. J Musculoskelet Neuronal Interact 4:325-334

28. Schoeffl I, Kemmler W, von Stengel S et al (2008) Physical activity, strength and VO2max have no significant influence on bone parameters in elderly women. J Musculoskelet Neuronal Interact 8:363-374

29. Fahrenberg J, Myrtek M, Wilk D, Kreutel K (1986) Multimodal assessment of life satisfaction: a study of patients with cardiovascular diseases. Psychother Psychosom Med Psychol 36:347-354

30. Bebenek M, Kemmler W, von Stengel S et al (2010) Effect of exercise and Cimicifuga racemosa (CR BNO 1055) on postmenopausal risk factors and complaints- the randomized controlled TRACE Study. Menopause 17:791-800

31. Kemmler W, Engelke K, Lauber D et al (2002) Exercise effects on fitness and bone mineral density in early postmenopausal women: 1-year EFOPS results. Med Sci Sports Exerc 34:2115-2123

32. R_Development_Core_Team (2020) R: A language and environment for Statistical Computing. R Foundation for Statistical Computing, Vienna, Austria

33. Honaker J, King G, Blackwell M (2011) Amelia II: a program for missing data. JSS 45:1-47

34. Barnard J, Rubin DB (1999) Small-sample degrees of freedom with multiple imputation. Biometrika 86:948-955

35. Hakkinen K, Alen M, Kallinen M et al (2000) Neuromuscular adaptation during prolonged strength training, detraining and restrength-training in middle-aged and elderly people. Eur J Appl Physiol 83:51-62

36. Ivey FM, Tracy BL, Lemmer JT et al (2000) Effects of strength training and detraining on muscle quality: age and gender comparisons. J Gerontol A 55:B152-157 (discussion B158-159)

37. Blocquiaux S, Gorski T, Van Roie E et al (2020) The effect of resistance training, detraining and retraining on muscle strength and power, myofibre size, satellite cells and myonuclei in older men. Exp Gerontol 133:110860

38. Kubo K, Ikebukuro T, Yata $\mathrm{H}$ et al (2010) Time course of changes in muscle and tendon properties during strength training and detraining. J Strength Cond Res 24:322-331

39. Lovell DI, Cuneo R, Gass GC (2010) The effect of strength training and short-term detraining on maximum force and the rate of force development of older men. Eur J Appl Physiol 109:429-435

40. Fiatarone MA, Marks EC, Ryan ND et al (1990) High-intensity strength training in nonagenarians. Effects on skeletal muscle. JAMA 263:3029-3034

41. Weineck J (2019) Optimales Training. Spitta-Verlag, Erlangen

42. Vuori I, Heinonen A, Sievanen H et al (1994) Effects of unilateral strength training and detraining on bone mineral density and content in young women: a study of mechanical loading and deloading on human bones. Calcif Tissue Int 55:59-67 
43. Eriksen EF (2010) Cellular mechanisms of bone remodeling. Rev Endocr Metab Disord 11:219-227

44. Shojaa N, von Stengel S, Schoene D et al (2020) Effect of exercise training on bone mineral density in postmenopausal women: a systematic review and meta-analysis of intervention studies. Front Physiol 11:1427-1444

45. Kemmler W, Kohl M, Frohlich M et al (2021) Detraining effects after 18 months of high intensity resistance training on osteosarcopenia in older men-six-month follow-up of the randomized controlled Franconian Osteopenia and Sarcopenia Trial (FrOST). Bone 142:115772

46. Snow CM, Williams DP, LaRiviere J et al (2001) Bone gains and losses follow seasonal training and detraining in gymnasts. Calcif Tissue Int 69:7-12

47. Saxon LK, Robling AG, Alam IM, Turner CH (2005) Mechanosensitivity of the rat skeleton decreases after a long period of loading, but is improved with time off. Bone 36:454-464

48. Kemmler W, Bebenek M, von Stengel S et al (2013) Effect of block-periodized exercise training on bone and coronary heart disease risk factors in early post-menopausal women: a randomized controlled study. Scand J Med Sci Sports 23:121-129
49. Kemmler W, Kohl M, von Stengel S (2016) Long-term effects of exercise in postmenopausal women: 16-year results of the Erlangen Fitness and Osteoporosis Prevention Study (EFOPS). Menopause 24:45-51

50. Kemmler W, Engelke K, von Stengel S (2016) Long-term exercise and bone mineral density changes in postmenopausal womenare there periods of reduced effectiveness? J Bone Miner Res $31: 215-222$

51. Sousa AC, Neiva HP, Gil MH et al (2020) Concurrent training and detraining: the influence of different aerobic intensities. J Strength Cond Res 34:2565-2574

52. Toraman NF (2005) Short term and long term detraining: is there any difference between young-old and old people? Br J Sports Med 39:561-564

Publisher's Note Springer Nature remains neutral with regard to jurisdictional claims in published maps and institutional affiliations. 